\title{
Neocortical Network Activity In Vivo Is Generated through a Dynamic Balance of Excitation and Inhibition
}

\author{
Bilal Haider, Alvaro Duque, Andrea R. Hasenstaub, and David A. McCormick \\ Department of Neurobiology, Kavli Institute for Neuroscience, Yale University School of Medicine, New Haven, Connecticut 06510
}

\begin{abstract}
The recurrent excitatory and inhibitory connections between and within layers of the cerebral cortex are fundamental to the operation of local cortical circuits. Models of cortical function often assume that recurrent excitation and inhibition are balanced, and we recently demonstrated that spontaneous network activity in vitro contains a precise balance of excitation and inhibition; however, the existence of a balance between excitation and inhibition in the intact and spontaneously active cerebral cortex has not been directly tested. We examined this hypothesis in the prefrontal cortex in vivo, during the slow $(<1 \mathrm{~Hz})$ oscillation in ketamine-xylazine-anesthetized ferrets. We measured persistent network activity (Up states) with extracellular multiple unit and local field potential recording, while simultaneously recording synaptic currents in nearby cells. We determined the reversal potential and conductance change over time during Up states and found that the body of Up state activity exhibited a steady reversal potential ( $-37 \mathrm{mV}$ on average) for hundreds of milliseconds, even during substantial ( $21 \mathrm{nS}$ on average) changes in membrane conductance. Furthermore, we found that both the initial and final segments of the Up state were characterized by significantly more depolarized reversal potentials and concomitant increases in excitatory conductance, compared with the stable middle portions of Up states. This ongoing temporal evolution between excitation and inhibition, which exhibits remarkable proportionality within and across neurons in active local networks, may allow for rapid transitions between relatively stable network states, permitting the modulation of neuronal responsiveness in a behaviorally relevant manner.
\end{abstract}

Key words: prefrontal cortex; Up states; intracellular; LFP; spontaneous activity; conductance

\section{Introduction}

The neurons of the cerebral cortex form a dense mosaic that contains horizontal and vertical connections traveling within and between the cortical layers (Szentagothai, 1978; Douglas and Martin, 2004). These connections are both convergent and divergent, such that the response of any individual neuron is determined by the influence of thousands of synaptic inputs (DeFelipe et al., 2002; Binzegger et al., 2004). The great majority of these inputs originate in local recurrent networks composed of both excitatory and inhibitory neurons. Furthermore, the state of these local networks in the intact brain is highly dynamic. Cortical neurons in vivo are constantly bombarded by synaptic inputs, resulting in a maintained depolarization during the waking state (Steriade et al., 2001; Chen and Fetz, 2005). Changes in these barrages of synaptic activity may strongly influence neuronal responsiveness (J. Anderson et al., 2000; Hô and Destexhe, 2000; Shu et al., 2003a; Brecht et al., 2004; Sachdev et al., 2004). Another consequence of this dense local recurrent connectivity is the ability of the cortex to generate persistent activity in the absence of sensory stimulation; this persistent neural firing has been particularly well studied in the prefrontal cortex (Fuster and Alexander,

Received Dec. 12, 2005; revised Feb. 16, 2006; accepted March 20, 2006.

This work was supported by the National Institutes of Health and the Kavli Foundation (D.A.M.), the Howard Hughes Medical Institute and the Beinecke Foundation (A.R.H.), and the Patricia S. Goldman-Rakic Fellowship from the Pfizer Corporation (B.H.). We thank Yousheng Shu and Robert Sachdev for helpful comments on this manuscript.

Correspondence should be addressed to David A. McCormick, Department of Neurobiology, Kavli Institute for Neuroscience, Yale University School of Medicine, 333 Cedar Street, New Haven, CT 06510. E-mail: david.mccormick@yale.edu.

DOI:10.1523/JNEUROSCI.5297-05.2006

Copyright $\odot 2006$ Society for Neuroscience $\quad$ 0270-6474/06/264535-11\$15.00/0
1971; Funahashi et al., 1989; Miller et al., 1996). Although computational models have often assumed that the influence of excitatory and inhibitory neurons must be approximately balanced in active cortical networks (van Vreeswijk and Sompolinsky, 1996; Shadlen and Newsome, 1998; Salinas and Sejnowski, 2000; Wang, 2001; Litvak et al., 2003), this hypothesis has not been directly examined in vivo.

One form of persistent neuronal activity that is readily studied, and which has yielded valuable information about the mechanisms and impact of spontaneous activity on neuronal responsiveness, is the cortical slow oscillation $(<1 \mathrm{~Hz})$ that occurs during natural sleep and certain forms of anesthesia (Metherate and Ashe, 1993; Steriade et al., 1993; Cowan and Wilson, 1994; Timofeev et al., 2000; Waters and Helmchen, 2004). The slow oscillation is characterized by rhythmic cycles of synaptically mediated depolarization and action potentials (Up states), followed by diminution of synaptic inputs, leading to membrane hyperpolarization and cessation of firing (Down states). Recently, we showed that recurrent network activity during Up states in vitro is generated and maintained by a precise balance between excitatory and inhibitory inputs (Sanchez-Vives and McCormick, 2000; Shu et al., 2003a) and that these periods of network activity may strongly influence neuronal responsiveness (McCormick et al., 2003; Shu et al., 2003b; Hasenstaub et al., 2005). Here, by examining intracellular synaptic potentials arriving during the Up state of the slow oscillation in vivo, we demonstrate that, indeed, excitatory and inhibitory conductances are proportional and well balanced during periods of spontaneous network activity in the intact neocortex; furthermore, both the initiation and cessation of network activity are associated with an increase in 
excitation. These results demonstrate that active local networks spontaneously maintain a depolarized membrane potential in which excitatory and inhibitory inputs remain dynamically balanced and that such periods of persistent activity may be initiated and terminated by excitatory influences.

\section{Materials and Methods}

Anesthesia and surgical preparation. Acute experiments were conducted on male ferrets (Mustela putorius furo), 2-4 months of age, weighing between 0.7 and $1.2 \mathrm{~kg}$. Animals were deeply anesthetized intramuscularly with a ketamine $(30 \mathrm{mg} / \mathrm{kg})$, xylazine $(1 \mathrm{mg} / \mathrm{kg})$ mixture, and supplemented intramuscularly approximately hourly to maintain a stable plane of surgical anesthesia throughout the experiments. Atropine $(0.5$ cc, s.c.) was administered to reduce secretions. Animals were lightly supplemented once with urethane $(1.5 \mathrm{ml}$, i.p.) in addition to the mixture described above. All incisions and pressure points were infiltrated with $3 \%$ lidocaine. Depth of anesthesia was assured by continuously monitoring electrocardiogram waveforms along with heart rate, respiration rate, and rectal body temperature, as well as by observing reactions to noxious stimuli (toe pinch) and reflexes. Our experiments required a deep level of anesthesia, and once recording commenced, anesthetic depth was also monitored by observation of intracortical field potentials, to ensure that the cortical slow oscillation was robustly expressed. Animals were placed in a stereotaxic apparatus, a midline incision was made, and the scalp, underlying fascia, and muscles were retracted. Bilateral craniotomies (2-3 $\mathrm{mm}$ in diameter) were performed $10-11 \mathrm{~mm}$ anterior to bregma, directly above the prefrontal cortex in the rostralmost portion of the anterior sigmoid gyrus and the dorsal orbital gyrus. A cisternal drainage was performed to relieve CSF pulsations. The dura overlying both hemispheres was dissected and retracted, and the craniotomies were filled with warm, sterile $4 \%$ agar. On completion of the experiments, animals were given a lethal dose of sodium pentobarbital intraperitoneally. All procedures were approved by the Yale University Animal Care and Use Committee and conform to the National Institutes of Health standards as recommended in Preparation and Maintenance of Higher Mammals During Neuroscience Experiments.

Recording and analysis. Tungsten microelectrodes $(0.3-0.5$ or 1-5 $\mathrm{M} \Omega$; Frederick Haer Company, Bowdoinham, ME) were used to record extracellular multiple units (MUs), or single units (SUs), along with local field potentials (LFPs). The raw signal was differentially amplified (A-M Systems 3000; A-M Systems, Sequim, WA) and recorded as a broadband $(0.1-20 \mathrm{kHz})$ signal, as well as being sent to two filters (Krohn-Hite, Brockton, MA) set at $300 \mathrm{~Hz}$ to $20 \mathrm{kHz}$, and $0.1-100 \mathrm{~Hz}$, for MU and LFP signals, respectively. The electrode was lowered into either the superficial or deep layers until robust multiple unit activity was detected, or until a well isolated SU was encountered. Extracellularly recorded single units were classified as fast spiking (FS) or regular spiking (RS) based on previously detailed criteria (McCormick et al., 1985; Hasenstaub et al., 2005). Intracellular electrodes were positioned to enter the cortex $200-$ $800 \mu \mathrm{m}$ away from the extracellular electrode. Intracellular electrodes contained either $2 \mathrm{M}$ potassium acetate, or $50 \mu \mathrm{M}$ lidocaine $N$-ethyl bromide (QX-314) and $2 \mathrm{M}$ cesium acetate to reduce or block $\mathrm{Na}^{+}$and $\mathrm{K}^{+}$ currents, as well as the h-current and $\mathrm{GABA}_{\mathrm{B}}$ synaptic potentials (Connors and Prince, 1982; Nathan et al., 1990; Nunez and Buno, 1992; Isaac and Wheal, 1993; Perkins and Wong, 1995). Intracellular electrodes filled with potassium acetate (used in current-clamp recordings) were beveled to have a final impedance of 65-100 $\mathrm{M} \Omega$, whereas electrodes filled with QX-314 and cesium acetate (used in voltage-clamp experiments) were pulled and beveled to have a lower series resistance, with impedances between 50 and $70 \mathrm{M} \Omega$. Current-clamp and single electrode voltageclamp (SEVC) recordings were performed with an AxoClamp 2B amplifier (Molecular Devices, Sunnyvale, CA). During SEVC protocols, switching frequency was at least $2 \mathrm{kHz}$, adequate amplifier gain and anti-alias filtering were used, and headstage output was continuously monitored on an oscilloscope to ensure adequate settling time. Extracellular broadband, LFP, MU, membrane potential $\left(V_{\mathrm{m}}\right)$, and current $(I)$ were sampled at 20,1,10,20, and $20 \mathrm{kHz}$, respectively, and digitized using the Spike2 System (Cambridge Electronics Design, Cambridge,
UK). Intracellularly recorded cells were classified as RS or FS according to standard criteria (McCormick et al., 1985; Nowak et al., 2003). Because of the blockade of action potentials during voltage-clamp recordings, when possible, electrophysiological identification of neurons was performed immediately on penetration of neurons shortly before action potentials became inactivated. Cells displaying IB (intrinsic bursting) properties were not included in the analyses. Additionally, recorded cells had to display robust $\mathrm{Up}$ states (at least $10 \mathrm{mV}$ in amplitude) and had to exhibit stable (Up state negative to $-50 \mathrm{mV}$ ) membrane potentials during the Up state with minimal ( 0 to $-0.1 \mathrm{nA}$ ) current injection. If identification was ambiguous, or the neuron failed any of the membrane potential criteria, it was not included in the analyzed population.

Intracellular records were segregated into Up/Down states in conjunction with the locally recorded MU network activity. Because our protocols inactivated spikes and required moving the membrane potential across the entire voltage range (in which synaptic depolarizations and hyperpolarizations become intermixed), we used the nearby MU recording as an indicator of the state of the local network. MU traces were down sampled, smoothed, and rectified, and two thresholds were set on this waveform. An upward crossing of the lower threshold signified a transition from the Down to the Up state. The waveform had to stay above the upper threshold for at least $300 \mathrm{~ms}$ to be counted as an Up state. When the waveform fell below the upper threshold, a transition to the Down state was indicated. The waveform then had to remain below the lower threshold for at least $100 \mathrm{~ms}$ to be counted as a Down state. These identified Up state membrane potential traces were graphically overlaid to ensure that the membrane potential trajectory at the starts and ends of Up states were aligned. In this manner, periods of transition could be separated from periods of stable network activity (Up states) or inactivity (Down states).

For voltage-clamp recordings, cells were progressively moved through a series of holding potentials, typically from -85 to $+10 \mathrm{mV}$, in steps of 5 or $10 \mathrm{mV}$, and held at each potential for a few minutes to record several $(>10)$ Up and Down states at each membrane potential. Holding potentials between -35 and -15 that exhibited $\mathrm{Ca}^{2+}$ spikes were discarded. We made no attempts to pharmacologically block voltage-gated $\mathrm{Ca}^{2+}$ channels. The method used here, which is based on work in other laboratories (Borg-Graham et al., 1998), has been used previously in our lab to calculate the conductance change during the Up state in vitro (Shu et al., 2003a). Briefly, average holding currents were constructed using at least $10 \mathrm{Up}$ states per holding potential, and used to construct current versus voltage $(I-V)$ plots (see Fig. 4). One line plotted the $I-V$ relationship during the Down state (baseline), whereas a second line plotted the $I-V$ relationship at some time point during the Up state. The point at which the Up and Down curves intersect was taken as the reversal potential [i.e., $E_{\mathrm{syn}}(t)$ ] for that particular point of the Up state. The $I-V$ plot for the Up state was constructed every millisecond, thereby giving the temporal evolution of the reversal potential throughout the Up state. We then assumed that the total measured current during the Up state was composed of two components: (1) the DC required for the desired holding potential and (2) the synaptic current $I_{\text {syn }}(t)$ flowing through the total synaptic conductance, $g_{\text {syn }}(t)$. The driving force of this current depends on two factors: the calculated reversal potential at time $(t)$, and the offsetcorrected membrane potential $V(t)$, giving the following: $I_{\text {syn }}(t)=g_{\text {syn }}$ $(t)\left[V(t)-E_{\text {syn }}(t)\right]$. Because we blocked a significant portion of voltagedependent intrinsic currents, we also assumed that the total measured conductance was dominated by the synaptic conductance, which was composed of the sum of the excitatory and inhibitory conductances as follows: $g_{\text {syn }}(t)=g_{\mathrm{e}}(t)+g_{\mathrm{i}}(t)$. Since $V=I_{\text {total }} / g_{\text {syn }}(t)$, then $E_{\text {syn }}(t)=$ $\left[g_{\mathrm{e}}(t) E_{\mathrm{e}}+g_{\mathrm{i}}(t) E_{\mathrm{i}}\right] /\left[g_{e}(t)+g_{\mathrm{i}}(t)\right]$. By assuming that the reversal potential for excitation $\left(E_{\mathrm{e}}\right)$ was $0 \mathrm{mV}$, and the reversal potential for inhibition $\left(E_{\mathrm{i}}\right)$ was $-75 \mathrm{mV}$ (as in Shu et al., 2003b), and by using the estimated reversal potentials calculated at time $(t)$ of the Up state, we could estimate the separate contributions of excitatory and inhibitory conductances at time point $(t)$. Solving for $g_{\mathrm{i}}(t)$ in the previous equation, and then substituting, we have the following:

$$
g_{\mathrm{i}}(t)=\frac{g_{\text {syn }}(t)\left[E_{\mathrm{e}}-E_{\mathrm{syn}}(t)\right]}{E_{\mathrm{e}}-E_{\mathrm{i}}} \text { and } g_{\mathrm{e}}(t)=g_{\text {syn }}(t)-g_{\mathrm{i}}(t) .
$$


Table 1. Intracellular properties of Up states in vivo

\begin{tabular}{|c|c|c|c|c|c|c|c|}
\hline & \multicolumn{7}{|c|}{ Parameters } \\
\hline & $\begin{array}{l}\text { RP mean } \\
(\mathrm{mV})\end{array}$ & $\begin{array}{l}\text { RPSD } \\
(\mathrm{mV})\end{array}$ & $\begin{array}{l}G \text { mean } \\
(\mathrm{nS})\end{array}$ & $\begin{array}{l}G \max \\
(\mathrm{nS})\end{array}$ & $\begin{array}{l}\text { G max time } \\
\text { (ms) }\end{array}$ & $\begin{array}{l}G_{e} \text { mean } \\
(n S)\end{array}$ & $\begin{array}{l}G_{\mathrm{i}} \text { mean } \\
(\mathrm{nS})\end{array}$ \\
\hline \multirow{2}{*}{\multicolumn{8}{|c|}{$\begin{array}{l}\text { Voltage clamp: } 0-500 \mathrm{~ms} \\
\text { from start of Up state }\end{array}$}} \\
\hline & & & & & & & \\
\hline Group $(n=8)$ & -37.17 & 6.54 & 21.14 & 25.99 & 105.87 & 9.24 & 11.41 \\
\hline SD & 7.97 & 3.30 & 10.62 & 23.19 & 37.16 & 4.13 & 8.42 \\
\hline \multirow[t]{3}{*}{ Range } & -49.14 & 0.95 & 9.37 & 13.08 & 54 & 6.68 & 4.00 \\
\hline & -24.53 & 7.96 & 49.98 & 66.47 & 152 & 17.34 & 33.70 \\
\hline & $\begin{array}{l}\text { RP mean } \\
(\mathrm{mV})\end{array}$ & $\begin{array}{l}\text { RPSD } \\
(\mathrm{mV})\end{array}$ & $\begin{array}{l}\text { G mean } \\
(\mathrm{nS})\end{array}$ & $\begin{array}{l}G \max \\
(\mathrm{nS})\end{array}$ & $\begin{array}{l}\text { G max time } \\
\text { (ms) }\end{array}$ & $\begin{array}{l}G_{e} \text { mean } \\
(n S)\end{array}$ & $\begin{array}{l}G_{\mathrm{i}} \text { mean } \\
(\mathrm{nS})\end{array}$ \\
\hline \multicolumn{8}{|c|}{$\begin{array}{c}\text { Voltage clamp: }-400 \text { to } 0 \mathrm{~ms} \\
\text { before end of Up state }\end{array}$} \\
\hline Group $(n=8)$ & -30.54 & 4.04 & 10.90 & 20.39 & -331.62 & 5.55 & 4.25 \\
\hline SD & 14.20 & 3.55 & 3.88 & 12.94 & 43.07 & 2.18 & 1.88 \\
\hline \multirow[t]{3}{*}{ Range } & -44.91 & 1.20 & 4.45 & 8.06 & -399 & 3.12 & 0.75 \\
\hline & -9.82 & 11.71 & 19.86 & 45.04 & -265 & 11.24 & 7.57 \\
\hline & $\begin{array}{l}\text { UP to DN } \\
(\mathrm{mV})\end{array}$ & $\begin{array}{l}\text { SD UP } \\
(\mathrm{mV})\end{array}$ & $\begin{array}{l}\text { SD DN } \\
(\mathrm{mV})\end{array}$ & $\begin{array}{l}\text { UP Dur } \\
\text { (s) }\end{array}$ & $\begin{array}{l}\text { DN Dur } \\
\text { (s) }\end{array}$ & $\begin{array}{l}\text { DN to UP } \\
\text { (s) }\end{array}$ & $\begin{array}{l}\text { UP to DN } \\
\text { (s) }\end{array}$ \\
\hline \multicolumn{8}{|l|}{ Current clamp } \\
\hline RS neurons $(n=9)$ & 13.19 & 2.13 & 0.79 & 1.58 & 0.26 & 0.13 & 0.17 \\
\hline SD & 4.64 & 0.61 & 0.29 & 0.51 & 0.17 & 0.04 & 0.03 \\
\hline \multirow[t]{2}{*}{ Range } & 9.30 & 1.55 & 0.25 & 0.76 & 0.16 & 0.07 & 0.13 \\
\hline & 18.59 & 3.44 & 1.16 & 2.48 & 0.41 & 0.17 & 0.21 \\
\hline FS neurons $(n=4)$ & 14.41 & 2.79 & 0.60 & 1.13 & 0.37 & 0.10 & 0.12 \\
\hline SD & 5.13 & 0.71 & 0.17 & 0.25 & 0.22 & 0.06 & 0.03 \\
\hline \multirow[t]{2}{*}{ Range } & 9.85 & 2.14 & 0.25 & 0.88 & 0.16 & 0.05 & 0.08 \\
\hline & 20.02 & 3.54 & 0.85 & 1.39 & 0.60 & 0.17 & 0.16 \\
\hline
\end{tabular}

$\mathrm{RP}$, Reverse potential; $G$, total conductance; $G_{\mathrm{e}}$, excitatory conductance; $G_{\mathrm{i}}$, inhibitory conductance; DN, Down state.
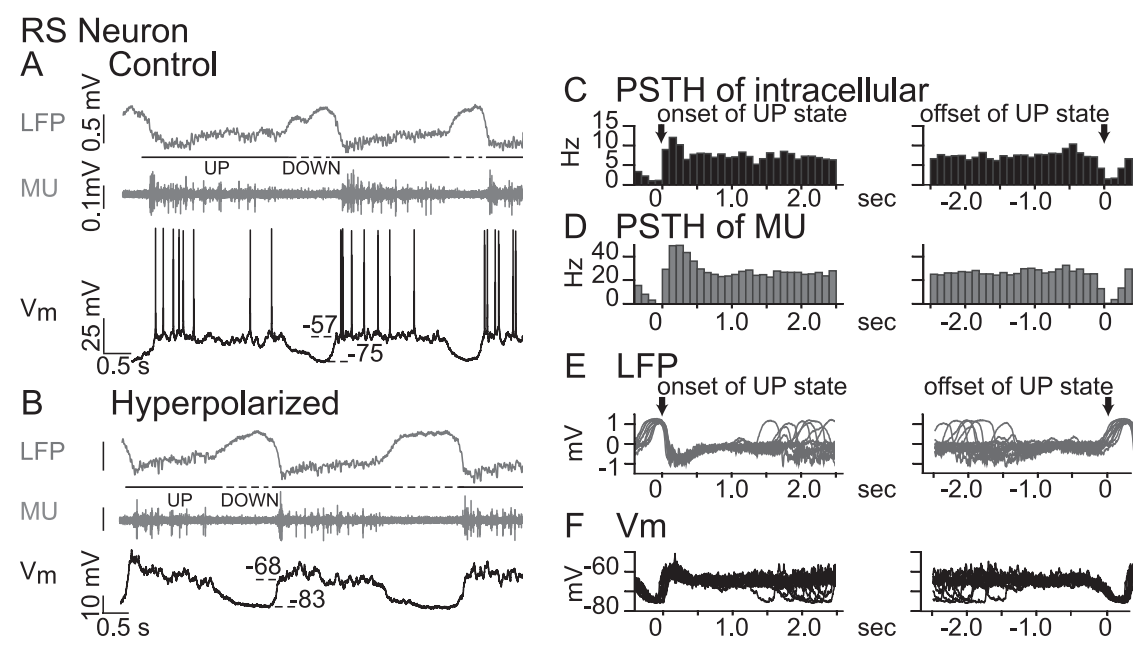

Figure 1. Spontaneous recurrent network activity in $\mathrm{RS}$ neurons of ferret prefrontal cortex in vivo. $\boldsymbol{A}$, Simultaneous LFP (top), extracellular MU (middle), and intracellular $\left(V_{m}\right)$ (bottom) recording reveals rhythmic cycle of local network activation (Up states; solid line), during which RS neurons become depolarized and fire action potentials simultaneous with the local network. Depolarization and action potential firing is followed by network quiescence (Down states; dashed line), during which membrane potential becomes hyperpolarized, and network activity ceases. $\boldsymbol{B}$, Hyperpolarization of the same RS neuron with negative DC reveals the synaptic barrages underlying the depolarizing envelope of the Up state ( $V_{m} ;$ bottom trace) that occur simultaneously with local network activity (middle and top; LFP and MU have same scale as in $A$ ). Distance between intracellular and extracellular recording was $<500 \mu \mathrm{m}$. C, PSTH constructed from the same cell as in $\boldsymbol{A}$ shows stable period of elevated spike rate during Up states. D, PSTH constructed from MU spikes recorded simultaneously as trials in $\boldsymbol{C}$ shows similar stable periods of network firing during Up states. $\boldsymbol{E}, \boldsymbol{F}$, Overlays of LFP and membrane potential of $>10$ Up states, aligned to the start and end of the Up state (arrows), reveal the rapid transitions, and the stable plateau of depolarization during Up states. Negative DC was injected to prevent spikes.
The estimates of conductance become unreliable at very low values (i.e., during the first and last few milliseconds of the Up state); for this reason, a threshold level $(\sim 10 \%)$ of synaptic conductance change (after subtraction of baseline input conductance) was set for each cell before decomposition into excitatory and inhibitory components (see Fig. 5E,F). Because the average length of the Up state in our recordings was $>1 \mathrm{~s}$ (Table 1 ), we focused our analysis on the first $500 \mathrm{~ms}$ of the Up state (synchronized on the beginning of the Up state) and the last $500 \mathrm{~ms}$ (synchronized on the onset of the subsequent Down state). Raw traces were digitally low-pass filtered at $1000 \mathrm{~Hz}$ in spike 2 before calculations were performed. Because individual recordings exhibited differing amounts of high-frequency membrane potential activity, derived excitatory and inhibitory conductances across the population were digitally low-pass filtered at $200 \mathrm{~Hz}$ before group data calculations; the contributions of these higher frequency components during the Up state have been detailed previously (Hasenstaub et al., 2005). All calculations and statistics were performed and plotted in MATLAB (The Mathworks, Natick, MA). Arithmetic means \pm SD are reported. All plots of population means are displayed along with the SEM, illustrated with dashed lines.

\section{Results}

Up states start and stop rapidly, synchronously, and exhibit stability in RS cells and the local network

To investigate the dynamics of persistently active cortical networks, we used simultaneous extracellular and intracellular recordings in the prefrontal cortex of ketamine-xylazine-anesthetized ferrets. Compared with some other forms of anesthesia, ketamine-xylazine produces robust, rhythmic slow oscillations, as shown in Figure 1. The recording configuration simultaneously shows the LFP, the MU activity in the local network, and the intracellular membrane potential $\left(V_{\mathrm{m}}\right)$ of a nearby electrophysiologically identified RS neuron [as described by McCormick et al. (1985) and Nowak et al. (2003)]. Note that the Up state (solid lines) is initiated by a highly synchronous cycle of depolarization in the intracellular trace simultaneous with local network MU firing and lowvoltage, high-frequency fluctuations in the LFP. This period of activity is followed by a rapid transition into the Down state (dashed lines), which is characterized by intracellular hyperpolarization simultaneous with cessation of network firing, and a large, low-frequency positive-going deflection in the LFP. During the Up state, the intracellular recording exhibits a steady and depolarized $(13.2 \pm 4.6 \mathrm{mV}$; $n=9$ ) (Table 1), yet fluctuating ("noisy") membrane potential (SD, $2.13 \pm 0.61 \mathrm{mV}$ ) 
(Table 1) crowned by irregular action potential discharge, both within and across Up states. Injection of hyperpolarizing current $(-0.1 \mathrm{nA})$ into the same neuron reveals the synaptic barrages underlying the Up state (Fig. $1 B$ ). Notice that the structure, frequency, and occurrence of membrane potential deviations are mostly unaltered by current injection and that these now subthreshold depolarizations still occur simultaneously with network activity.

The relationship between local network activity and intracellular events is shown by overlaying traces of intracellular membrane potential and the LFP, and also by quantifying single-cell and network behavior over time with peristimulus time histograms (PSTHs) that are triggered on the onset and offset of many $(>10) \mathrm{Up}$ states (Fig. $1 C-F$ ). The left panels are all aligned to the onset of the Up state (Fig. $1 C$, left arrow), whereas the right panels are aligned to the offset of the Up state (Fig. $1 C$, right arrow). The same neuron as in Figure $1 A$ was allowed to spike spontaneously, and a PSTH was constructed for the intracellularly measured spikes, as well as for the spikes recorded extracellularly from the local network. The average firing rates of the single cell $(\sim 8 \mathrm{~Hz})$ and of the cells in the local network are remarkably stable throughout the Up state, and both single and multiple units rapidly and simultaneously transition into the Up state (Fig. $1 C, D$ ). The rapid transition from the Down state into the Up state occurs synchronously throughout the local network ( $128.5 \pm 44.6 \mathrm{~ms} ; n=9$ cells for $>10 \mathrm{Up}$ states/cell) (Table 1). This transition, from quiescence into a stable pattern of activity, occurs at a significantly faster rate than the transition into the Down state $(173.9 \pm 28.3 \mathrm{~ms} ; n=9$ cells for $>10 \mathrm{Up}$ states/cell; $T_{\text {transition in }}<T_{\text {transition out }} ; p<0.05$; paired $t$ test) (Table 1 ). Additionally, note the slight increase in firing rate for this RS cell toward the end of the Up state. Figure 1,E and F, contains overlays of many simultaneously recorded LFPs and intracellular membrane potentials, respectively. The intracellular membrane potential is hyperpolarized to prevent action potentials and reveal synaptic barrages. It can be seen that maintenance of the Up state, both in the membrane potential and in the local network activity, is remarkably stable across time. Again, transitions into the stable plateau of depolarization are rapid and well correlated with local network activity. This correlation is visible across time, when, for example, short-duration Up states in the intracellular traces are paralleled by equally short-duration Up states as seen in the LFP.

Up states start and stop rapidly, synchronously, and exhibit stability in FS interneurons and the local network

The relationship of local network Up states to the activity of physiologically identified FS interneurons was also examined (Fig. 2). These interneurons fire trains of high-frequency action potentials in response to brief current pulses (data not shown) and show little or no firing rate adaptation, as previously described (McCormick et al., 1985; Nowak et al., 2003). FS neurons are both depolarized to a similar degree as the RS cells (14.4 \pm 5.1 $\mathrm{mV} ; n=4$ cells for $>10 \mathrm{Up}$ states/cell) (Table 1 ) and fire action potentials simultaneous with MU firing and LFP activity. In no case did we observe FS interneurons firing action potentials out of phase with the local network. The injection of hyperpolarizing current into FS interneurons reveals the structure of synaptic barrages arriving simultaneously with activity in the local network (Fig. 2 B). Although we often observed that the variability of the membrane potential during the Up state in FS cells (SD, $2.79 \pm 0.71 \mathrm{mV} ; n=4$ cells for $>10 \mathrm{Up}$ states/cell) (Table 1 ) was greater than in RS cells, this difference was not significant in our population ( $t$ test; $p>0.05$ ). The transition rate of the membrane potential into the Up state in FS interneurons was not significantly different from the transition out of the Up state $(t$ test; $p>$ $0.05)$.

The initiation, sustenance, and termination of Up states in FS interneurons (Fig. 2C-F) follow network dynamics similar to those seen in pyramidal cells (compare with Fig. $1 C-F$ ). The PSTH of spontaneous action potentials shows that the intracellular and simultaneously recorded MU spike rates rise and fall together (Fig. 2C,D). Additionally, these PSTHs demonstrate that the average firing rates of single interneurons and those of the local network are robust and relatively stable during the body of the Up state, although the FS interneuron firing rate slowly decreases during the last $200 \mathrm{~ms}$ of the Up state (Fig. 2C). Simultaneous recordings of the LFP (Fig. $2 E$ ) and membrane potential show that the depolarization of FS interneurons and the increase in local network occurs rapidly and synchronously. Additionally, it can be seen that the membrane potential of FS interneurons remains remarkably stable and elevated during the course of the Up state ( $>10$ overlays) (Fig. $2 F)$.

Intracellular synaptic barrages recorded during Up states are a mixture of excitation and inhibition

To dissect the contributions of these simultaneously active excitatory and inhibitory circuits, both current-clamp and voltageclamp intracellular recordings were performed in RS pyramidal neurons during Up states. To better isolate these synaptic poten- 

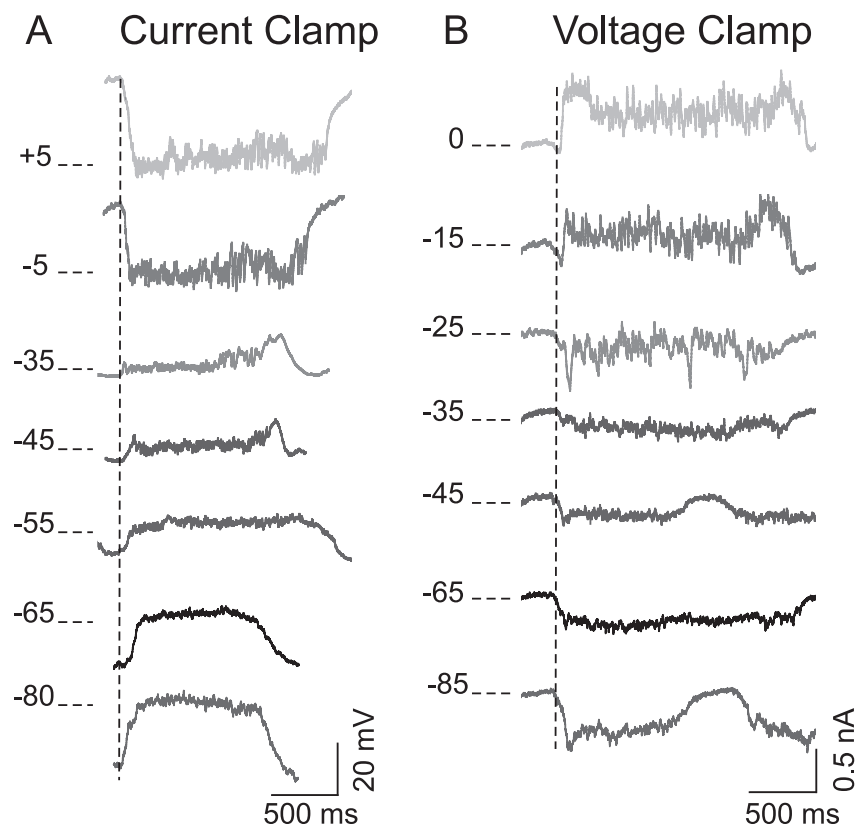

Figure 3. Both excitatory and inhibitory synaptic barrages occur during Up states. $\boldsymbol{A}$, In current-clamp mode, hyperpolarized membrane potential values ( $-80 \mathrm{mV}$; bottom trace) show depolarizations during Up states, whereas depolarized membrane potential values $(+5$ $\mathrm{mV}$; top trace) reveal hyperpolarizations during Up states. Intermediate potentials (middle traces) exhibit a mixture of depolarizing and hyperpolarizing synaptic activity. The membrane potential labels refer to those achieved at the peak of the Up state. $\boldsymbol{B}$, SEVC reveals synaptic currents at the soma that underlie Up states. Negative holding potentials ( $-85 \mathrm{mV}$; bottom trace) reveal inward currents during Up states, whereas positive holding potentials ( $0 \mathrm{mV}$; top trace) show outward currents during Up states. A mixture of excitatory and inhibitory currents is present at intermediate holding potentials ( -35 to $-25 \mathrm{mV}$; middle traces).

tials, we recorded in conditions that minimize the contributions of intrinsic voltage-dependent conductances (see Materials and Methods). In this manner, the membrane potential of RS neurons (electrophysiologically classified before complete blockade of $\mathrm{Na}^{+}$and $\mathrm{K}^{+}$currents) can be moved to many levels via intracellular current injection, and with confidence that the majority of recorded activity is attributable to synaptic conductances. While blocking or reducing intrinsic voltage-dependent $\mathrm{K}^{+}$and $\mathrm{Na}^{+}$conductances, current-clamp recordings (Fig. $3 A$ ) reveal that the Up state is simultaneously composed of both excitatory and inhibitory synaptic potentials. Hyperpolarizing the cell with the intracellular injection of DC such that the Up state is near the reversal potential for GABAergic chloride-mediated inhibition (Fig. $3 A,-80 \mathrm{mV}$, bottom trace) predominantly isolates depolarizing (glutamatergic) influences on the membrane potential occurring during the Up state. Conversely, depolarizing the cell with DC injection such that the Up state is near the expected reversal potential for glutamatergic excitation (Fig. $3 A,-5$ to +5 $\mathrm{mV}$, top traces) reveals the GABAergic chloride-mediated hyperpolarizing membrane potentials occurring during the Up state. From these recordings, it can be seen that the membrane potential during the Up state exhibits a mixture of excitatory and inhibitory barrages. As seen in Figure $3 A$, when we use constant current to depolarize the neuron to near $-35 \mathrm{mV}$, the membrane potential exhibits little net deflection from the Down state on initiation of the Up state. Negative to this reversal potential, membrane potential deflections are depolarizing during the Up state, whereas at more positive values, the membrane potential deflections are hyperpolarizing during the Up state.

To determine the synaptic currents underlying these mem-
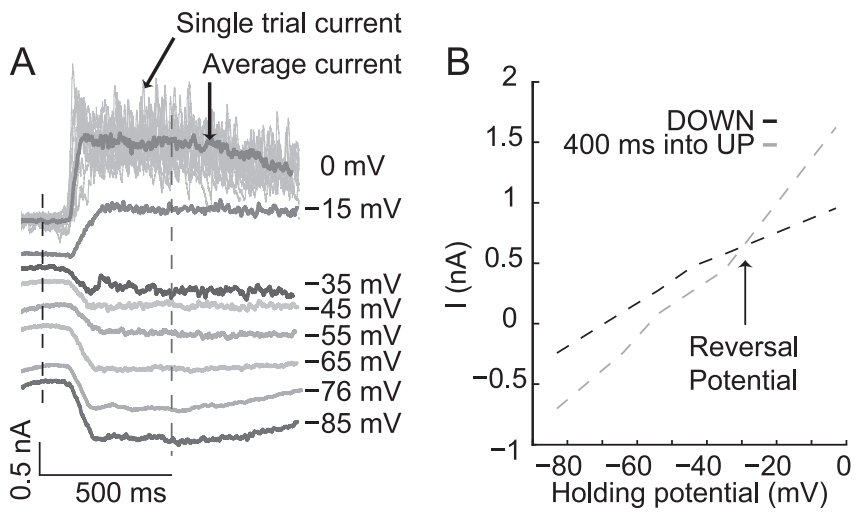

Figure 4. Method for estimation of synaptic reversal potential during Up states. $\boldsymbol{A}$, Single neurons are progressively moved through a series of holding potentials at which many successive Up states are recorded (single trial current; top arrow) and aligned to the starts of Up states to produce the average Up state evoked current (average current; bottom arrow) for each holding potential. $\boldsymbol{B}$, Current-voltage plots across the range of holding potentials are constructed for both Down state (black dashed line) and for each point during the Up state (e.g., 400 ms after Up start; gray dashed line). The intersection point of the two I-V plots represents the reversal potential for the Up state at that particular time.

brane potential deflections recorded at the soma, we performed SEVC, again while blocking or reducing $\mathrm{Na}^{+}, \mathrm{K}^{+}$, and other conductances by including QX-314 and $\mathrm{Cs}^{+}$in the recording electrode. We have demonstrated previously, with similar pharmacological measures and recording techniques, that the average synaptic currents arriving at the soma [Shu et al. (2003a), their supplemental information] can be accurately measured in this manner, keeping in mind the known limitations of voltage-clamp experiments in extensively branched structures (Spruston et al., 1993; Schaefer et al., 2003). Thus, holding cells at a command potential that is near the reversal potential for GABAergic chloride-mediated inhibition (Fig. $3 B,-85 \mathrm{mV}$ ), reveals predominantly inward EPSCs, whereas holding cells near the reversal potential for glutamate-mediated excitation will predominantly isolate outward IPSCs (Fig. $3 B, 0 \mathrm{mV}$ ). Because both inhibitory and excitatory currents are arriving simultaneously during the Up state, their combined influence will exhibit a "reversal potential" at which point the net driving force for this mixture of excitatory and inhibitory currents, as recorded at the soma, is minimal.

\section{Estimating reversal potential of synaptic barrages during Up states}

To determine the temporal relationship of excitation and inhibition in active local networks, we constructed plots of the currentvoltage relationship across a wide voltage range, and measured the reversal potential and conductance change over the evolution of the Up state. Synaptic currents elicited by a minimum of $10 \mathrm{Up}$ states were recorded in SEVC at each holding potential. The resulting current measurements were aligned to the start of the Up state (see Materials and Methods) and averaged to yield a mean current evoked from the Up state at each holding potential (Fig. $4 A, 0 \mathrm{mV}$, single trial and average traces, top arrows). Two plots were then constructed: one series of points came from current measurements at each holding potential during the Down state (Fig. 4A, $B$, black dashed line), whereas the second set of points represented the current measurements at each holding potential, for a given time point during the body of the Up state (Fig. $4 A, B$, gray dashed line). $I-V$ plot construction (polynomial fit) from the $I-V$ values recorded during the Up state was iterated, milli- 
second by millisecond, from the start of the Up state, whereas the Down state $I-V$ plot was always constructed from the same set of (baseline) points. The intersection of these two $I-V$ plots represents the reversal potential for the synaptic barrages of the Up state at that particular time point, whereas the change in slope of the curves around the reversal point represents the change in total membrane conductance.

\section{Reversal potential is stable and excitatory and inhibitory conductances are proportional and balanced during the Up state}

In this manner, synaptic currents were recorded across the entire voltage range (Fig. 5A), and, assuming linear summation of currents at the soma (see Materials and Methods), the estimated reversal potential at each time point of the Up state was determined. Figure $5 C$ shows the reversal potential calculated using the above-described method and aligned on the onset of the Up state. The Up state reversal potential starts off at very depolarized levels (indicating a predominance of excitation) but then quickly reaches a stable and maintained level throughout the body of the Up state (mean, $-38.3 \pm 1.2 \mathrm{mV}$ from 100 to $500 \mathrm{~ms}$ ). This estimate of the time-varying reversal potential allows deconstruction of the total conductance of the Up state into an estimate of the underlying excitatory and inhibitory conductances (see Materials and Methods). It can be seen (Fig. $5 E$ ) that the excitatory conductance $\left(g_{\mathrm{e}}\right)$ initially leads at the start of the Up state, followed by rapid engagement of the inhibitory conductance $\left(g_{\mathrm{i}}\right)$, and thereafter, the two conductances remain at an elevated yet stable level throughout the Up state.

Similarly, the time-varying reversal potential can be calculated for the same neuron and aligned on the offset of the Up state (Fig. $5 D)$. Again, the neuron has a steady reversal potential $(-34.5 \pm$ 1.2 , from -500 to $-250 \mathrm{~ms}$ ) throughout this relatively stable portion of the Up state. Figure 5, E and $F$, shows that both $g_{\mathrm{e}}$ and $g_{\mathrm{i}}$ rise and fall in a proportional manner, whereas the total conductance remains steady through the body of the Up state; however, as the Up state begins to collapse ( $\sim 250 \mathrm{~ms}$ before Up end), a predominance of excitatory conductance emerges. This is reflected in the progressively more depolarized reversal potential estimate toward the end of the Up state in Figure 5D.

Additional examination of excitatory and inhibitory conductances during network activity confirmed this significant increase in the dominance of excitatory conductance toward the end of the Up state. The average excitatory and inhibitory conductances calculated for each neuron were normalized to peak conductance, and then combined and aligned to either the onset (Fig. $5 G$ ) or the offset (Fig. $5 H$ ) of the Up state. The ratio of excitatory to inhibitory conductance in the population exhibits a significant increase, for $\sim 150 \mathrm{~ms}$, before the collapse of the Up state (Fig. $5 H)\left(n=8 ;-301\right.$ to $-147 \mathrm{~ms}$ before Up end; $G_{\mathrm{e}}>G_{\mathrm{i}}$; paired Wilcoxon sign rank test; $p<0.01$ ). Conversely, the excitatory and inhibitory conductances aligned at the beginning of the Up state remain relatively balanced, although there is a small but significant tendency for excitatory conductance to dominate inhibition at the very beginning of the Up state $\left(0-10 \mathrm{~ms} ; G_{\mathrm{e}}>G_{\mathrm{i}}\right.$; paired Wilcoxon sign rank test; $p<0.05$ ) (data not shown).

\section{Excitation dominates the initiation and termination of network activity}

We reasoned that if these changes in measured conductance occurring toward the end of the Up state were reflective of patterns of excitatory and inhibitory synaptic bombardment, then a similar relationship should be evident by examining the firing rates of
A

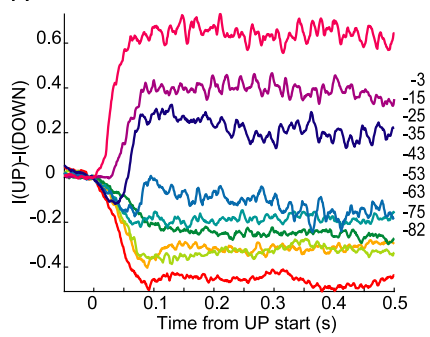

B
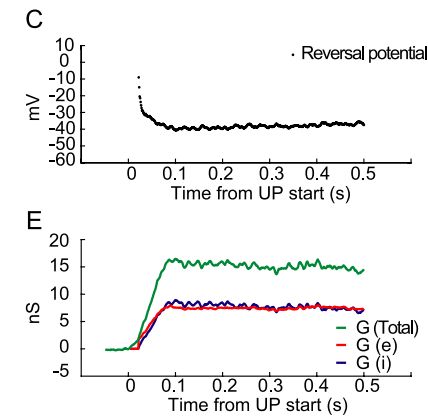

G

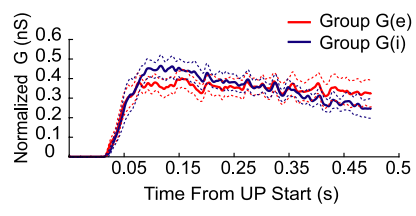

Figure 5. Currents evoked during Up states exhibit a steady reversal potential. $A$, Average synaptic currents recorded for $500 \mathrm{~ms}$ and aligned to the start of the Up state, for many different holding potentials. $\boldsymbol{B}$, Synaptic currents recorded at various holding potentials, from $500 \mathrm{~ms}$ before the end ( $0 \mathrm{~ms}$ ) of the Up state. Baseline current is subtracted in both $\boldsymbol{A}$ and $\boldsymbol{B}$. C, Plot of reversal potential over time, calculated from current-voltage relationships in the first $500 \mathrm{~ms}$, shows a steady reversal potential during the course of the Up state $(-38.3 \pm 1.16 \mathrm{mV}$ from 100 to $500 \mathrm{~ms})$. D, Similar plot of reversal potential over time, aligned to end of the Up state, exhibits a stable value $(-34.5 \pm 1.24$ from -500 to $-250 \mathrm{~ms})$ before the collapse of the Up state. Note depolarized reversal potentials at both initiation and cessation of Up state. $\boldsymbol{E}$, Total conductance change evoked from Down to Up transition (mean $g_{\text {Total }}, 15.0 \pm 1.0 \mathrm{nS}$ ) remains steady during the course of the Up state. Excitatory and inhibitory conductances rise and fall together and remain steady during the Up state (mean $g_{\mathrm{e}}, 7.4 \pm 0.4 \mathrm{nS}$; mean $g_{\mathrm{i}}, 7.7 \pm 0.7 \mathrm{nS}$ ). $\boldsymbol{F}$, Similar plot aligned on end of the Up state shows that total, excitatory, and inhibitory conductances remain steady until the Up state begins to collapse (from -500 to $-250 \mathrm{~ms}$; mean $g_{\text {Total, }} 11.7 \pm 0.6 \mathrm{nS}$; mean $g_{\mathrm{e}^{\prime}} 6.3 \pm 0.2 \mathrm{nS} ; g_{\mathrm{i}}, 5.4 \pm 0.4 \mathrm{nS}$ ). Note relative increase in $g_{\mathrm{e}}$ toward the end of Up state. $G$, Population $(n=8)$ excitatory $\left(G_{\mathrm{e}} ;\right.$ solid red line) and inhibitory $\left(G_{i}\right.$; solid blue line) conductances aligned on start of the Up state and calculated for $500 \mathrm{~ms}$ thereafter. $G_{\mathrm{e}}$ and $G_{\mathrm{i}}$ for each neuron normalized to peak $G_{\text {total }}$ in that cell. Excitation leads at the beginning, inhibition is rapidly engaged, and then the two remain balanced thereafter. The dashed lines indicate SEM. $\boldsymbol{H}$, Similar group plot of $G_{\mathrm{e}}$ and $G_{\mathrm{i}}$ aligned on the end of the Up state. $G_{\mathrm{e}}$ and $G_{i}$ normalized to peak $G_{\text {total }}$ for each neuron. $G_{\mathrm{e}}$ is significantly greater than $G_{\mathrm{i}}$ for $\sim 150$ ms before Up state collapse ( -301 to -147 ms before Up end; $G_{e}>G_{i}$; paired Wilcoxon sign rank test; $p<0.01$ ).

RS and FS cell types. Action potentials recorded extracellularly from electrophysiologically identified FS $(n=6)$ and RS $(n=6)$ cell types (Hasenstaub et al., 2005) were used to construct PSTHs ( $>50$ Up states per neuron; 25 ms bins) triggered on the starts and ends of the Up state. Individual histograms were normalized to peak firing rate (mean RS firing rate, $6.7 \pm 2.4 \mathrm{~Hz}$; mean FS firing rate, $24.7 \pm 9.1 \mathrm{~Hz}$ ) and then combined to yield a population average of RS and FS cell firing rates at the start and end of the Up state (Fig. 6A,B) (plot convolved with normalized Gaussian filter SD of 0.5 for graphical purpose). These recordings revealed that RS neurons maintain significantly elevated spiking activity, for $200 \mathrm{~ms}$ before the end of the Up state, compared with 

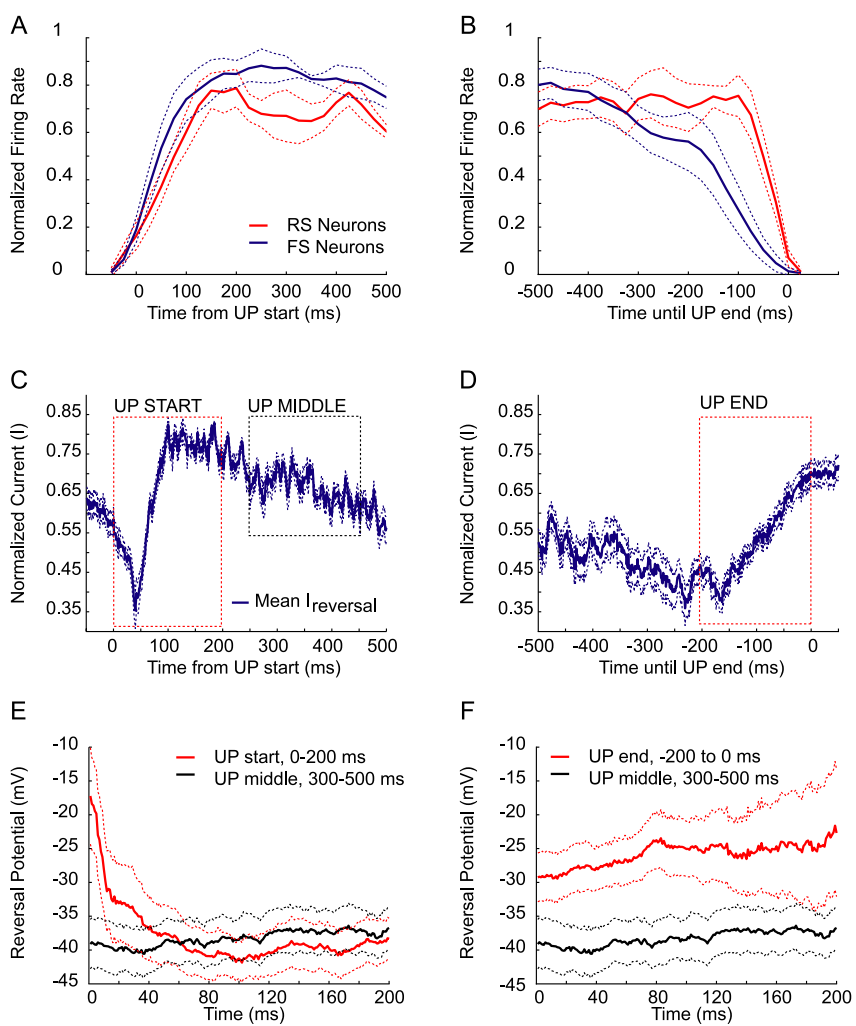

Figure 6. Excitation dominates both the beginning and end of Up states. $\boldsymbol{A}$, Peak normalized population firing rates of extracellularly recorded and electrophysiologically identified RS (thick red line; $n=6$ ) and FS (thick blue line; $n=6$ ) neurons, aligned on the start of the Up state ( $>50 \mathrm{Up} \mathrm{states/neuron;} \mathrm{RS}=6.7 \pm 2.4 \mathrm{~Hz} ; \mathrm{FS}=24.7 \pm 9.1 \mathrm{~Hz}$ before normalization). The dashed lines indicate SEM. $\boldsymbol{B}$, Similar plot as $\boldsymbol{A}$ aligned on the end of the Up state. Normalized RS firing is significantly elevated compared with FS firing for $200 \mathrm{~ms}$ before Up end ( -200 to $0 \mathrm{~ms}$ before Up end; RS $>$ FS; paired Wilcoxon sign rank test; $p<0.01$ ). $C$, Average synaptic currents recorded when the membrane potential is held near the reversal potential of the body of the Up state, traces aligned on the start of the Up state. Peak normalized average currents (thick blue line) recorded at the two holding potentials immediately adjacent to the calculated reversal potential for each neuron $(n=5)$. Near reversal potential, the Up state is clearly dominated by excitation for the first $50 \mathrm{~ms}$ before the current swings toward outward. The dashed lines represent the SEM. D, Similar plot to $\boldsymbol{C}$, aligned at the end of the Up state. Note the increase in inward excitatory current preceding transition from Up to Down. $E$, Population average reversal potential for same neurons as in $\boldsymbol{C}$ and $\boldsymbol{D}$, calculated for the first $200 \mathrm{~ms}$ from Up onset (Up start; C, red box), and compared against the reversal potential calculated from 300-500 ms (Up middle; $\boldsymbol{C}$, black box) into the Up state. The reversal potential from 0 to 43 ms of the Up state (red trace) is significantly more depolarized than the reversal potential (black trace) during the body of the Up state (paired Wilcoxon sign rank test; $p<0.01$ ). The dashed lines represent the SEM. $\boldsymbol{F}$, Similar plot as $\boldsymbol{E}$ but aligned to the end of the Up state. Population average reversal potential calculated for last $200 \mathrm{~ms}$ preceding collapse of the Up state (Up end; $\boldsymbol{D}$, red box), compared with $200 \mathrm{~ms}$ from the middle of the Up state, same as in $\boldsymbol{E}$. Reversal potential from -200 to $0 \mathrm{~ms}$ preceding Up end (red trace) is significantly more depolarized than segment from the stable middle portion (black trace) of the Up state (paired Wilcoxon sign rank test; $p<0.01$ ).

the firing of FS neurons (Fig. $6 \mathrm{~B})\left(-200\right.$ to $0 \mathrm{~ms}$; $\mathrm{RS}_{\text {end }}>\mathrm{FS}_{\text {end }}$; paired Wilcoxon sign rank test; $p<0.01)$. These normalized firing rates did not differ significantly during the body of the Up state nor at the start of the Up state (paired Wilcoxon sign rank test; $p>0.1$ ). Importantly, these firing patterns of RS and FS cells (recorded extracellularly to ensure minimal disturbance of the spontaneous behavior of the neurons) are temporally consistent with the intracellular conductance measurements (compare with Fig. 5H).

Because the estimate of excitatory and inhibitory conductance requires a certain threshold amount of net current change (see Materials and Methods), our estimates of excitation and inhibi- tion typically began a few milliseconds after the initiation of the Up state and ceased a few milliseconds before the end of the Up state (Fig. $5 E, F$ ). Thus, to more precisely investigate the temporal dynamics of intracellular excitation and inhibition nearer to the initiation and cessation points of the Up state, we averaged the raw Up state current traces, recorded continuously $(>10 \mathrm{Up}$ states) from each of the two holding potentials immediately adjacent to the calculated reversal potential for each individual neuron. These average currents were normalized to the peak current at each potential, for each neuron, and then combined to yield a population average of evoked currents near the reversal potential of the Up state. Importantly, these averages did not rely on any a priori threshold or assumptions about the data. In this way, we found that the initiation of the Up state (Fig. 6C) is clearly dominated by an inward shift in the current, which is followed, after $\sim 50 \mathrm{~ms}$, by a shift in the average current toward outward $(n=5$ neurons). These same population traces were also aligned to the end of the Up state, where they show the slow shift toward inward current before the end of the Up state (Fig. 6D). The calculated reversal potentials for the first (Fig. $6 C$, Up start, red box), middle (Fig. 6C, Up middle, black box), and last $200 \mathrm{~ms}$ of the Up state (Fig. 6D, Up end, red box) were then aligned in time and compared for these neurons. As suggested by the raw current traces, the average population reversal potential was significantly more depolarized for the first $43 \mathrm{~ms}$ of the Up state (Fig. $6 E$, red trace) compared with the middle portion of the Up state (Up start $>\mathrm{Up}$ middle; paired Wilcoxon sign rank test; $p<0.01$ ). This period of dominant excitatory current falls well within the average transition time from the Down state to the Up state (Table 1). Additionally, the average reversal potential for these same data revealed that the last $200 \mathrm{~ms}$ of the Up state (Fig. 6 F, red trace) was significantly more depolarized than the middle $200 \mathrm{~ms}$ of the Up state (Fig. $6 D$, black trace) (Up end $>$ Up middle; paired Wilcoxon sign rank test; $p<0.01)$. Thus, although excitation and inhibition are proportional during the stable portions of recurrent network activity, asymmetry exists, both in firing properties and in intracellular conductances, at the initiation and the termination of stable periods of network activity.

\section{Excitation and inhibition maintain proportionality during ongoing network activity}

Having examined the initiation and termination points of the Up state, we next investigated the interrelationship of excitation and inhibition during the long, stable portions of recurrent network activity by plotting the excitatory versus inhibitory conductances over time. Single neurons show a remarkably linear and stable relationship between excitatory and inhibitory synaptic conductances calculated over the course of many hundreds of milliseconds of the Up state (Fig. 7A) (Up start to $500 \mathrm{~ms}$ ). Following the progression of the Up state through time (indicated by movement from warmer to cooler hues in the plot) demonstrates that the excitatory and inhibitory conductances within a cell maintain a stable ratio (slope of linear fit, $m=0.98 ; r^{2}=0.78$ ) despite large changes in total conductance $\left(g_{\max }=22.7 \mathrm{nS}\right)$. Note that the excitatory conductance leads at the beginning (red-orange), whereupon inhibition is rapidly engaged (yellow), followed by balanced activity during the Up state. Thus, importantly, the activation of inhibitory circuits during the Up state is not expressed as a simple transient disynaptic feedforward inhibition, but rather scales continuously with the level of ongoing activity in the local network. Recall that the LFP moves negative during Up states, whereas during Down states the LFP moves positive (Figs. $1 A, 2 A)$. Over many (>30) sequential Up states, IPSC intensity 
A

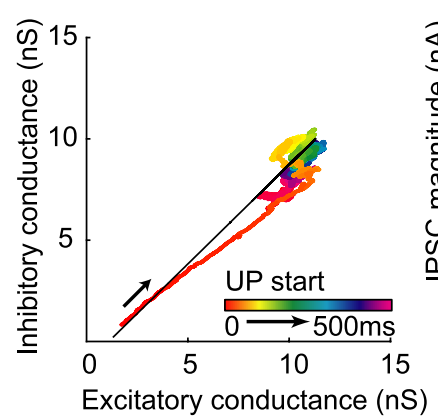

B

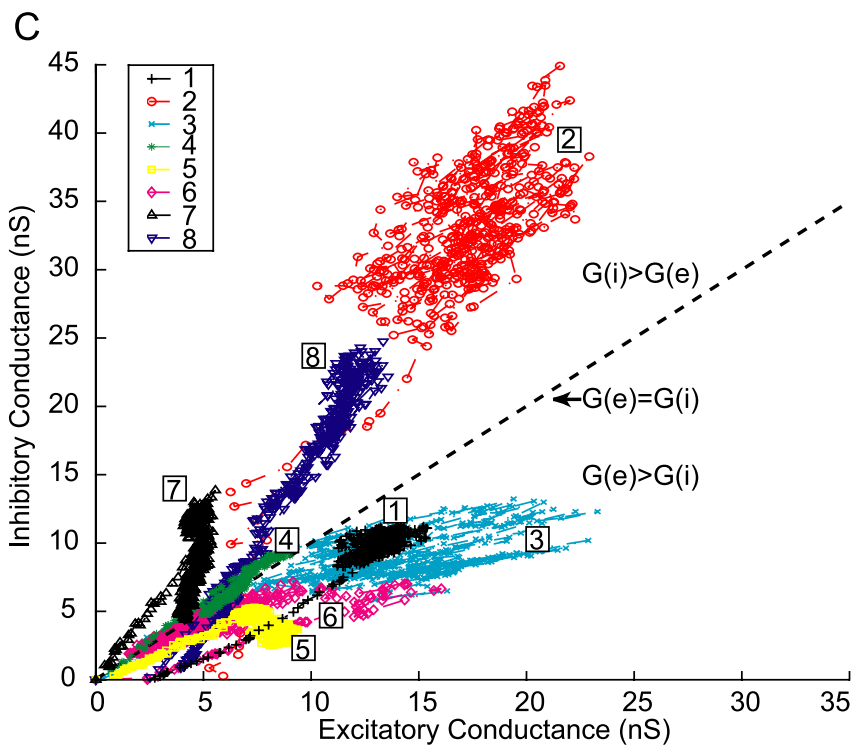

Figure 7. Excitatory and inhibitory conductances are proportional and balanced during Up states. $\boldsymbol{A}$, Plot of calculated excitatory and inhibitory conductances in a single neuron during the course of the Up state $(0-500 \mathrm{~ms}$, indicated by progressive movement through color bar) shows that excitation and inhibition remain proportional and nearly equal despite large changes in total conductance (slope of linear fit, $m=0.98 ; r^{2}=0.78$ ). Note that the start of the Up state shows a deviation toward excitation, but rapidly swings toward inhibition and thereafter exhibits a balance between the two. $\boldsymbol{B}$, Scatterplot of IPSC magnitude versus the amplitude of the nearby $(<500 \mu \mathrm{m})$ LFP during the course of the Up state. The intensity of IPSCs mirrors the intensity of population activity $\left(r^{2}=-0.81\right)$ over time. Correlation is negative since downward deflections of the LFP indicate network activation. C, Excitation and inhibition are proportional and balanced both within and across neurons during recurrent network activity. Scatterplot of excitatory versus inhibitory conductances for a population of neurons $(n=8)$, calculated for $500 \mathrm{~ms}$ from the start of the Up state. Note the linear relationship for each individual neuron, as well as the clustering around a ratio of equal excitatory and inhibitory conductances $\left(G_{\mathrm{e}}=G_{\mathrm{i}}\right.$; dashed line; 4 of 8 cells biased toward excitation, 3 of 8 cells toward inhibition, 1 of 8 cells approximately equal; population reversal potential, $-37.2 \pm 6.5 \mathrm{mV}$ ).

mirrors the intensity of neuronal population activity as represented by the LFP (Fig. $7 B)\left(r^{2}=-0.81\right)$. This relatively linear relationship of IPSC intensity to the LFP is also observed when comparing the IPSC amplitude with the magnitude of MU activity recorded nearby (data not shown). This indicates that increasing excitatory drive in the local network results in a rapid and proportional activation of inhibitory currents within single neurons during the body of the Up state.

Although the precise ratio of excitation to inhibition is unique for each neuron, there is a remarkable linearity for this relationship, both within single neurons (Fig. 7C, as indicated by color and number), and across the entire population $(n=8)$ during recurrent network activity. The majority of neurons cluster around a ratio of equal excitation and inhibition (dashed line), with individual cells showing biases toward either excitation (four of eight cells; below dashed line) or inhibition (three of eight cells; above dashed line). Nonetheless, the average reversal potential in the population during this time period (500 ms) was $-37.2 \pm 6.5 \mathrm{mV}$, indicating a nearly equal contribution for excitation and inhibition ( -37.5 being halfway between our values for $E_{\mathrm{e}}=0 \mathrm{mV}$ and $\left.E_{\mathrm{i}}=-75 \mathrm{mV}\right)$. This proportionality for $G_{\mathrm{e}}$ and $G_{i}$ within the population of neurons was exhibited for the duration of the stable portion of the Up state, despite $21.1 \pm 10.6 \mathrm{nS}$ of average conductance change (Table 1).

\section{Discussion}

Here, we have shown that the conductance in pyramidal cells caused by spontaneous network activity in vivo is remarkably well balanced between excitation and inhibition, and that this proportionality is maintained and remains stable during fluctuations in total membrane conductance. This proportionality is the result of the interaction between recurrent excitation and feedback inhibition, which scales with the level of activity present in the local network. This stable, balanced activity keeps neurons at a noisy and elevated level of depolarization near their firing threshold.

\section{Synaptic conductance measurements in vivo}

The conductance values we have measured here in vivo are in agreement with the values we have previously measured in vitro (Shu et al., 2003a) and are in agreement with other studies (J. S. Anderson et al., 2000; Wehr and Zador, 2003). Contrary to recent reports asserting that inhibitory conductances are twofold to threefold larger than excitatory conductances during spontaneous activity in vivo (Rudolph et al., 2005), we find that our excitatory and inhibitory conductance estimates, derived from isolated synaptic currents recorded across a large voltage range, exhibit, on average, nearly equal proportionality.

Our measurements reflect the cumulative contributions of excitation and inhibition arriving at proximal portions of the neuron, and, importantly, near the action potential initiation site (Stuart et al., 1997). Our measurements, like all somatic recordings, cannot make rigorous estimates of synaptic events in the distal dendrites. Our conductance estimates are ratios of the overall excitatory and inhibitory drive contained in the local network, and this precise mixture of currents-arriving at the soma-will determine spike initiation. It is likely that unique distributions of ligand and voltage-gated channels affect the ratio of excitatory and inhibitory conductances in more distal compartments (Migliore and Shepherd, 2002). Moreover, somatically recorded estimates of distal synaptic events may be affected by the nonlinear characteristics of pyramidal cell dendrites (Spruston et al., 1993; Schaefer et al., 2003). However, by using both QX-314 and $\mathrm{Cs}^{+}$in our recording solution, we increase $R_{\mathrm{m}}$ and attenuate the decay of excitatory currents spreading from the proximal dendrites, which receive the majority of excitatory inputs in neocortex (Peters, 2002), allowing our measures to include more of the total excitatory synaptic current arriving in the neuron as a whole. Because inhibitory synapses dominate excitatory synapses in perisomatic regions, our measures may be biased toward inhibition. Indeed, this somatic dominance of inhibition may explain previous suggestions that inhibitory conductances are much larger than the excitatory conductances ( $\mathrm{Ru}$ dolph et al., 2005). Additionally, our measures of the variance of the membrane potential during the Up state (on segments of data with no action potentials and which contain no state transitions) (see Materials and Methods), are significantly 
lower than previously estimated (Rudolph et al., 2005). Correctly determining the change in membrane conductance and level of membrane variance during network activity is critical to understanding how background activity modulates neuronal responsiveness (Shu et al., 2003a,b).

Another difficulty with previous attempts to measure the change in conductance associated with Up states is that they have been based on a limited portion of the $I-V$ relationship. We find that extrapolating the reversal potential from limited portions of the $I-V$ curve (between rest and action potential threshold) may yield significantly different estimates of membrane conductance change and reversal potential than results based on examination of the entire voltage range (Fig. $4 B$ ). Although our experiments required blockade of $\mathrm{K}^{+}$currents, which normally contribute to the resting membrane potential, recordings without pharmacological blockade [Shu et al. (2003a), their supplemental Fig. 3] indicate that the synaptic reversal potential is nonetheless approximately balanced between excitation and inhibition. Moreover, although there is variability in the exact ratio of excitation to inhibition for each individual cell, the population as a whole shows a strong tendency toward a nearly equal ratio. Remarkably, once this particular proportion of excitation and inhibition (which determines the synaptic reversal potential) is established, this ratio (i.e., the slope of each cell in Fig. 7C) is maintained despite rapid changes in membrane conductance attributable to ongoing activity. Differences in the ratio are likely the result of neurons receiving a varying complement of excitatory and inhibitory connections. Previous reports have detailed the relationship of cortical cell types and the slow oscillation (Contreras and Steriade, 1995, 1997; Contreras et al., 1996), and our results extend these findings to the conductances underlying network activity. Although computational studies have shown that altering the spatiotemporal properties of excitatory and inhibitory synapses can affect somatic potentials (Koch et al., 1983; Koch, 1999), the unique contributions of excitation and inhibition based on location, cell type, and connectivity remain to be further explored.

\section{Functional implications of ongoing spontaneous activity}

Cortical neurons are subjected to constant synaptic bombardment, as observed in a variety of species and preparations (Cowan and Wilson, 1994; Arieli et al., 1995; Sanchez-Vives and McCormick, 2000; Steriade et al., 2001; Cossart et al., 2003). Importantly, intracellular recordings from awake animals suggest a significant level of ongoing activity that maintains the membrane potential of cortical neurons in a noisy and depolarized state (Steriade et al., 2001; Chen and Fetz, 2005). A consequence of synaptic bombardment is a conductance load that decreases the membrane time constant, requiring more frequent or more synchronized inputs to affect the membrane potential (Softky and Koch, 1993); we have demonstrated previously that the high-frequency activity and synchronization we observe in active cortical networks can have significant effects on spike timing during spontaneous activity (Hasenstaub et al., 2005) (for review, see Singer and Gray, 1995; Buzsaki and Draguhn, 2004). This robust and synchronized activity is contrary to reports of Up states recorded in rat barrel cortex in vivo (in whole-cell patch clamp), in which the majority of Up state activity was subthreshold (firing rate, $\sim 0.5 \mathrm{~Hz}$ ) (Petersen et al., 2003; Brecht et al., 2004) (but see Sachdev et al., 2004). It is unlikely that the intracellular activity we observe is a consequence of damage, as we see compa- rable levels of activity from extracellularly isolated single units (Fig. 6), and, importantly, in whole-cell recordings during Up states in vitro (Hasenstaub et al., 2005). More likely, these disparities result from differences in species, cortical area, layer, or choice of anesthetic. It is worth noting that primate prefrontal neurons engaged in behavioral tasks show markedly elevated firing rates (Constantinidis and Goldman-Rakic, 2002), similar to the elevated firing rates we see in ferret prefrontal neurons. Although recent studies have debated the exact interaction of background activity with sensory information, (Azouz and Gray, 1999; J. Anderson et al., 2000; Petersen et al., 2003; Sachdev et al., 2004), it remains a critically important issue to determine the baseline state of cortical network activity, and to determine whether it is spatiotemporally sparse or robust, both spontaneously and after sensory stimulation (deCharms and Zador, 2000; Olshausen and Field, 2004).

\section{Dynamic interaction between excitation and inhibition}

Finally, our results demonstrate that both the initiation and cessation of the Up state are accompanied by significantly more excitation than the stable, middle portions of the Up state. The time course of excitation is remarkably different at the beginning versus the end of the Up state: feedforward excitation initiates the transition into the Up state, followed by rapid feedback inhibition, and maintenance of the membrane potential in a stable configuration near firing threshold (see Fig. 5), whereas the end of the Up state is preceded by a slowly emerging dominance of excitatory current. Previous observations in vitro have demonstrated that recurrent activity can be terminated by excitatory influences (Shu et al., 2003a). Additionally, activity-dependent membrane currents have been implicated in termination of network activity (Compte et al., 2003), and we presume that synaptic depression or other activity-dependent mechanisms also contribute. Recently, human EEG studies have demonstrated that cortical activity during the slow oscillation exhibits characteristics of a traveling wave, as we have previously shown in vitro (Sanchez-Vives and McCormick, 2000), and that a strong source of this oscillation in humans is in the frontal cortex (Massimini et al., 2004); moreover, neocortical pyramidal cells send and receive a significant amount of long-range excitatory connections, whereas the majority of interneuron output is restricted to the local circuit (McGuire et al., 1991). Thus, it may be the case that an increase in excitation arriving toward the end of the Up state, via long-range excitatory connections from regions now experiencing the crest of a traveling wave, drives a critical population of excitatory neurons simultaneously into refractoriness, whereupon local recurrent activity collapses (Gutkin et al., 2001).

Our observations suggest that local networks in vivo can be rapidly activated by excitatory synaptic barrages that proportionally activate feedback inhibition, the balance of which keeps neurons in a dynamically depolarized state that can confer significant computational advantages to both the probability and the timing of action potentials (Hô and Destexhe, 2000; Shu et al., 2003b; Hasenstaub et al., 2005). Moreover, long-range excitatory connectivity may provide a means for selectively "biasing" the state of local networks. The modulation of ongoing, balanced local network activity may provide a powerful and rapid mechanism to control the excitability and responsiveness of interconnected regions of the cortex. Such cellular mechanisms of local network activity may be operating in awake animals to rapidly control the gain of neuronal responsiveness in a behaviorally relevant manner. 


\section{References}

Anderson J, Lampl I, Reichova I, Carandini M, Ferster D (2000) Stimulus dependence of two-state fluctuations of membrane potential in cat visual cortex. Nat Neurosci 3:617-621.

Anderson JS, Carandini M, Ferster D (2000) Orientation tuning of input conductance, excitation, and inhibition in cat primary visual cortex. J Neurophysiol 84:909-926.

Arieli A, Shoham D, Hildesheim R, Grinvald A (1995) Coherent spatiotemporal patterns of ongoing activity revealed by real-time optical imaging coupled with single-unit recording in the cat visual cortex. J Neurophysiol 73:2072-2093.

Azouz R, Gray CM (1999) Cellular mechanisms contributing to response variability of cortical neurons in vivo. J Neurosci 19:2209-2223.

Binzegger T, Douglas RJ, Martin KAC (2004) A quantitative map of the circuit of cat primary visual cortex. J Neurosci 24:8441-8453.

Borg-Graham LJ, Monier C, Fregnac Y (1998) Visual input evokes transient and strong shunting inhibition in visual cortical neurons. Nature 393:369-373.

Brecht M, Schneider M, Sakmann B, Margrie TW (2004) Whisker movements evoked by stimulation of single pyramidal cells in rat motor cortex. Nature 427:704-710.

Buzsaki G, Draguhn A (2004) Neuronal oscillations in cortical networks. Science 304:1926-1929.

Chen D, Fetz EE (2005) Characteristic membrane potential trajectories in primate sensorimotor cortex neurons recorded in vivo. J Neurophysiol 94:2713-2725.

Compte A, Sanchez-Vives MV, McCormick DA, Wang XJ (2003) Cellular and network mechanisms of slow oscillatory activity $(<1 \mathrm{~Hz})$ and wave propagations in a cortical network model. J Neurophysiol 89:2707-2725.

Connors BW, Prince DA (1982) Effects of local anesthetic QX-314 on the membrane properties of hippocampal pyramidal neurons. J Pharmacol Exp Ther 220:476-481.

Constantinidis C, Goldman-Rakic PS (2002) Correlated discharges among putative pyramidal neurons and interneurons in the primate prefrontal cortex. J Neurophysiol 88:3487-3497.

Contreras D, Steriade M (1995) Cellular basis of EEG slow rhythms: a study of dynamic corticothalamic relationships. J Neurosci 15:604-622.

Contreras D, Steriade M (1997) State-dependent fluctuations of lowfrequency rhythms in corticothalamic networks. Neuroscience 76:25-38.

Contreras D, Timofeev I, Steriade M (1996) Mechanisms of long-lasting hyperpolarizations underlying slow sleep oscillations in cat corticothalamic networks. J Physiol (Lond) 494:251-264.

Cossart R, Aronov D, Yuste R (2003) Attractor dynamics of network UP states in the neocortex. Nature 423:283-288.

Cowan RL, Wilson CJ (1994) Spontaneous firing patterns and axonal projections of single corticostriatal neurons in the rat medial agranular cortex. J Neurophysiol 71:17-32.

deCharms RC, Zador A (2000) Neural representation and the cortical code. Annu Rev Neurosci 23:613-647.

DeFelipe J, Alonso-Nanclares L, Arellano JI (2002) Microstructure of the neocortex: comparative aspects. J Neurocytol 31:299-316.

Douglas RJ, Martin KA (2004) Neuronal circuits of the neocortex. Annu Rev Neurosci 27:419-451.

Funahashi S, Bruce CJ, Goldman-Rakic PS (1989) Mnemonic coding of visual space in the monkey's dorsolateral prefrontal cortex. J Neurophysiol 61:331-349.

Fuster JM, Alexander GE (1971) Neuron activity related to short-term memory. Science 173:652-654.

Gutkin BS, Laing CR, Colby CL, Chow CC, Ermentrout GB (2001) Turning on and off with excitation: the role of spike-timing asynchrony and synchrony in sustained neural activity. J Comput Neurosci 11:121-134.

Hasenstaub A, Shu Y, Haider B, Kraushaar U, Duque A, McCormick DA (2005) Inhibitory postsynaptic potentials carry synchronized frequency information in active cortical networks. Neuron 47:423-435.

Hô N, Destexhe A (2000) Synaptic background activity enhances the responsiveness of neocortical pyramidal neurons. J Neurophysiol 84:1488-1496.

Isaac JT, Wheal HV (1993) The local anaesthetic QX-314 enables enhanced whole-cell recordings of excitatory synaptic currents in rat hippocampal slices in vitro. Neurosci Lett 150:227-230.

Koch C (1999) Synaptic interactions in a passive dendritic tree. In: Biophys- ics of computation: information processing in single neurons, pp 117141. New York: Oxford UP.

Koch C, Poggio T, Torre V (1983) Nonlinear interactions in a dendritic tree: localization, timing, and role in information processing. Proc Natl Acad Sci USA 80:2799-2802.

Litvak V, Sompolinsky H, Segev I, Abeles M (2003) On the transmission of rate code in long feedforward networks with excitatory-inhibitory balance. J Neurosci 23:3006-3015.

Massimini M, Huber R, Ferrarelli F, Hill S, Tononi G (2004) The sleep slow oscillation as a traveling wave. J Neurosci 24:6862-6870.

McCormick DA, Connors BW, Lighthall JW, Prince DA (1985) Comparative electrophysiology of pyramidal and sparsely spiny stellate neurons of the neocortex. J Neurophysiol 54:782-806.

McCormick DA, Shu Y, Hasenstaub A, Sanchez-Vives M, Badoual M, Bal T (2003) Persistent cortical activity: mechanisms of generation and effects on neuronal excitability. Cereb Cortex 13:1219-1231.

McGuire BA, Gilbert CD, Rivlin PK, Wiesel TN (1991) Targets of horizontal connections in macaque primary visual cortex. J Comp Neurol 305:370-392.

Metherate R, Ashe JH (1993) Ionic flux contributions to neocortical slow waves and nucleus basalis-mediated activation: whole-cell recordings in vivo. J Neurosci 13:5312-5323.

Migliore M, Shepherd GM (2002) Emerging rules for the distributions of active dendritic conductances. Nat Rev Neurosci 3:362-370.

Miller EK, Erickson CA, Desimone R (1996) Neural mechanisms of visual working memory in prefrontal cortex of the macaque. J Neurosci 16:5154-5167.

Nathan T, Jensen MS, Lambert JD (1990) The slow inhibitory postsynaptic potential in rat hippocampal CA1 neurones is blocked by intracellular injection of QX-314. Neurosci Lett 110:309-313.

Nowak LG, Azouz R, Sanchez-Vives MV, Gray CM, McCormick DA (2003) Electrophysiological classes of cat primary visual cortical neurons in vivo as revealed by quantitative analyses. J Neurophysiol 89:1541-1566.

Nunez A, Buno W (1992) Intracellular effects of QX-314 and Cs ${ }^{+}$in hippocampal pyramidal neurons in vivo. Exp Neurol 115:266-270.

Olshausen BA, Field DJ (2004) Sparse coding of sensory inputs. Curr Opin Neurobiol 14:481-487.

Perkins KL, Wong RK (1995) Intracellular QX-314 blocks the hyperpolarization-activated inward current Iq in hippocampal CA1 pyramidal cells. J Neurophysiol 73:911-915.

Peters A (2002) Examining neocortical circuits: some background and facts. J Neurocytol 31:183-193.

Petersen CC, Hahn TT, Mehta M, Grinvald A, Sakmann B (2003) Interaction of sensory responses with spontaneous depolarization in layer $2 / 3$ barrel cortex. Proc Natl Acad Sci USA 100:13638-13643.

Rudolph M, Pelletier JG, Pare D, Destexhe A (2005) Characterization of synaptic conductances and integrative properties during electrically induced EEG-activated states in neocortical neurons in vivo. J Neurophysiol 94:2805-2821.

Sachdev RN, Ebner FF, Wilson CJ (2004) Effect of subthreshold up and down states on the whisker-evoked response in somatosensory cortex. J Neurophysiol 92:3511-3521.

Salinas E, Sejnowski TJ (2000) Impact of correlated synaptic input on output firing rate and variability in simple neuronal models. J Neurosci 20:6193-6209.

Sanchez-Vives MV, McCormick DA (2000) Cellular and network mechanisms of rhythmic recurrent activity in neocortex. Nat Neurosci 3:1027-1034.

Schaefer AT, Helmstaedter M, Sakmann B, Korngreen A (2003) Correction of conductance measurements in non-space-clamped structures: 1 . Voltage-gated $\mathrm{K}^{+}$channels. Biophys J 84:3508-3528.

Shadlen MN, Newsome WT (1998) The variable discharge of cortical neurons: implications for connectivity, computation, and information coding. J Neurosci 18:3870-3896.

Shu Y, Hasenstaub A, McCormick DA (2003a) Turning on and off recurrent balanced cortical activity. Nature 423:288-293.

Shu Y, Hasenstaub A, Badoual M, Bal T, McCormick DA (2003b) Barrages of synaptic activity control the gain and sensitivity of cortical neurons. J Neurosci 23:10388-10401.

Singer W, Gray CM (1995) Visual feature integration and the temporal correlation hypothesis. Annu Rev Neurosci 18:555-586.

Softky WR, Koch C (1993) The highly irregular firing of cortical cells is 
inconsistent with temporal integration of random EPSPs. J Neurosci 13:334-350.

Spruston N, Jaffe DB, Williams SH, Johnston D (1993) Voltage- and spaceclamp errors associated with the measurement of electrotonically remote synaptic events. J Neurophysiol 70:781-802.

Steriade M, Nunez A, Amzica F (1993) A novel slow ( $<1 \mathrm{~Hz}$ ) oscillation of neocortical neurons in vivo: depolarizing and hyperpolarizing components. J Neurosci 13:3252-3265.

Steriade M, Timofeev I, Grenier F (2001) Natural waking and sleep states: a view from inside neocortical neurons. J Neurophysiol 85:1969-1985.

Stuart G, Schiller J, Sakmann B (1997) Action potential initiation and propagation in rat neocortical pyramidal neurons. J Physiol (Lond) 505:617-632.

Szentagothai J (1978) The Ferrier Lecture, 1977. The neuron network of the cerebral cortex: a functional interpretation. Proc R Soc Lond B Biol Sci 201:219-248.

Timofeev I, Grenier F, Bazhenov M, Sejnowski TJ, Steriade M (2000) Origin of slow cortical oscillations in deafferented cortical slabs. Cereb Cortex 10:1185-1199.

van Vreeswijk C, Sompolinsky H (1996) Chaos in neuronal networks with balanced excitatory and inhibitory activity. Science 274:1724-1726.

Wang XJ (2001) Synaptic reverberation underlying mnemonic persistent activity. Trends Neurosci 24:455-463.

Waters J, Helmchen F (2004) Boosting of action potential backpropagation by neocortical network activity in vivo. J Neurosci 24:11127-11136.

Wehr M, Zador AM (2003) Balanced inhibition underlies tuning and sharpens spike timing in auditory cortex. Nature 426:442-446. 